\title{
Road Traffic Modeling using Data Communication Networks
}

\author{
Mohamed S. Abd Alzaher \\ Electronics and Communication \\ Engineering department, Faculty of \\ Engineering, University of Ain Shams \\ 1, El Sarayat street Abbassia, Cairo, \\ Egypt
}

\author{
Hussein A. Elsayed \\ Electronics and Communication \\ Engineering department, Faculty of \\ Engineering, University of Ain Shams \\ 1, El Sarayat street Abbassia, Cairo, \\ Egypt
}

\author{
Somaia I. Kayed \\ Electronics and Communication \\ Engineering department, Faculty of \\ Engineering, University of Ain Shams \\ 1, El Sarayat street Abbassia, Cairo, \\ Egypt
}

\author{
Wagdy R. Anis \\ Electronics and Communication Engineering department, \\ Faculty of Engineering, University of Ain Shams \\ 1, El Sarayat street Abbassia, Cairo, Egypt
}

\begin{abstract}
Nowadays the world became filled by several vulnerable problems that need to a quick solution and occupies the thinking of most people. From these problems the Road Network traffic crises that produces congestion, collision and ... etc, thus a lot of issues are effected, such that working times, people psychology and so on. So we need to find an effective solution under the world financial crisis circumstances and surrounding environment, means that a solution achieve low cost, where from the suggested solutions for reducing or facilitation the traffic motion problems mainly are depending on the mobile communication (cell phone, GPS, Satellite... etc.), but it need to more funding. We find that the Data Network can perform the wanted aim due to the fast development nowadays of the computer systems and technologies, where we look forward to reach to certain model based on complete mapping between all Road Network parameters and the corresponding in the Data Network. This paper will discuss a mapping between Road Network parameters and Data Network parameters that will be used to get the model.
\end{abstract}

\section{Keywords}

Data Networks, Road Traffic, Switches, Routers and Network Protocols.

\section{INTRODUCTION}

Firstly let's know what is traffic? This question represents general meaning, where it latent in the Road Network Traffic and Data Network Traffic, each of them will be described below. The traffic is the amount of sent and received objects by visitors, whether Road Network (Vehicles) or Data Network Packet Data Unit (PDU). According to the wide scene between the Data Network and Road Network, we refer to the mapping between them, the challenge was the notion of joining two different fields in one object, this due to dependency of one to the other, because of needing to reach to better future, where always the development should convey the needs of people, that is contained for example in the roads traffic roads, transportation methods and .... etc. So the several Data networks methods problems and their multiple solutions that drive us to crystallize it to be appropriate for using to road network crisis and being the Road Network problem represent a boatel neck for reaching to pacific traversing world, there is no dogmatic solution for the critical problem. We will be derived towards a real problem along the world due to gigantic vehicle production within fixed Road environment, the own model will need to a flexible simulation that can be matched or accomplished in the statistic results. Therefore by getting alternate paths for the congested paths of the Road we can control the Road Network events.

\section{ROAD NETWORKS}

Recently the Road Network became represent very important issue in our society, where no one can dispense to it, within several ages, but nowadays the Road network and its transportation methods have a conflict due to non generated roads and non synchronous with the different vehicle size. Let's discuss each Road network Parameter, its effect in the traffic motions such congestion and same statistics. The studies proved that the congestion occurs at lowest level (Level F), means that the vehicles speed reach to their minimum value, then appear the Stop and Go condition phenomenon and vehicle stop within 60 seconds, also may occurs temporary waiting that cause (Shock Wave). Nowadays the average number of vehicles becomes near to the average number of people, within the environment circumstances the request size increased about the exhibited or available roads according to the congestion components (Roads, Vehicles and Walkers). Also the cross-sections affect greatly, where it may cause traffic jam so the studies proved that the Cross-Section saturated at $512 \mathrm{C}, \mathrm{C} \ldots$ actual gate Width by meter and the Gate Width $=2.5: 9 \mathrm{~m}$, for example when a driver go to certain Cross-section to return back the opposite side may cause a queue because of the number of returned vehicles rather than available time of traffic light green sign and also the waited vehicles in the middle lane that need to go to the opposite direction. The lane capacity road connections $=1800: 2000$ vehicle/hour (i.e. average 1900 Vehicle/Hour), the platform capacity $=\mathrm{aX}+\mathrm{bX}^{2}, \mathrm{X} \ldots$ the walker speed $1 \mathrm{~m} / \mathrm{min}$, $\mathrm{a}$ and $\mathrm{b}$ are constants related to the actual platform width by meter. 
According to the several vehicle kinds and the resulted problems due to the surrounded challenges such that size vs. service and available speed, we find that the private vehicle has a special performance in form brakes, speed acceleration and dodging and the vice versa proportional to the huge vehicles such Bus and trailer, they are controlled by multiple limits and conditions, where they occupies (3:4 doubles) of the private vehicle size. The purpose of improving the traffic efficiency proportional to the moving vehicles (Crash reduction, Congestion reduction, Delay reduction, Noise reduction and Pollution reduction) where they are solved by the following systems (Unidirectional system, Return avoidance, Movable Barriers, Pedestrian Management, Public transportation regulation and Traffic signs, signals or Traffic Light), note that the standing vehicles due to (Loading, Unloading or Parking). There is a statistic of Missouri State road network accidents or crashes caused by ages, as shown in figure 1. ${ }^{[5]}$

From the methods of Increasing Traffic Capacity:

\subsection{Low Capacity Methods}

Round size controlling of the cross-section, Wait controlling at least before or after cross, Appling the unidirectional system that increase the efficiency with $15 \%$, Improving the traffic light performance, Engineering preliminary stage of the traffic axes and Traffic awareness \{ Traffic engineering, Traffic roles, Traffic behaviors training and Transporting the expertise to the traffic field\}.

\subsection{Medium Capacity Methods}

Remap the roads and cross-sections, Redesign the traffic lights to minimize the delay time, Join the traffic lights by a central control system and expand the road width.

\subsection{High Capacity Methods}

Construct unidirectional bridge at high traffic regions, Construct a generic cross-section, Construct new axes inside the city and Construct sequential bridges to make smooth traffic flow, Thus from the proposed solutions.

- Equivalent vehicle unit with a specific size or coordinates for Community Transportation.

- Adding extra fees for the vehicles their uses the crowded roads.

According to the several vehicle kinds and the resulted problems due to the surrounded challenges such that size vs. service and available speed, we find that the private vehicle has a special performance in form brakes, speed acceleration and dodging and the vice versa proportional to the huge vehicles such Bus and trailer, they are controlled by multiple limits and conditions, where they occupies (3:4 doubles) of the private vehicle size.

The purpose of improving the traffic efficiency proportional to the moving vehicles (Crash reduction, Congestion reduction, Delay reduction, Noise reduction and Pollution reduction) where they are solved by the following systems (Unidirectional system, Return avoidance, Movable Barriers, Pedestrian Management,
Public transportation regulation and Traffic signs, signals or Traffic Light), note that the standing vehicles due to (Loading, Unloading or Parking).

There is a statistic of Missouri State road network accidents or crashes caused by ages, as shown in figure $1 .{ }^{[5]}$

\section{DATA NETWORKS}

There are three categories acquainted with the own target:

\subsection{Data Network Components ${ }^{[6]}$.}

\subsubsection{Repeater}

Without the repeaters will occur distortion and noise is added to the signal along the transmission line, There are two types of the repeater (Generative such in above figure and Non-Generative), firstly the Generative repeater extracts the data and then retransmit again but after single clock cycle, where it higher cost because of producing synchronization signals between the Transmitter and Receiver, so high processing is achieved, secondary the Non-Generative Repeater is only amplifier but generates both of signal and noise so it is lower cost; the SNR is obtained in the following figure of the three cases No-Repeater, Non-Generative repeater and Generative Repeater.

\subsubsection{Hub}

Is a Layer one device or network device not communication device, where can understand layer one (physical layer) so it can deal with line coding such Manchester and Differential Manchester but not layer two (Data Link) so can't d understand MAC address, also is called Garbage on Garbage out (GIGO), makes cross-over and considered one collision domain because its connection physically star but logically bus.

\subsubsection{Switch}

Is a Layer two device, it can understand the Data Link layer (MAC address), but not Physical Layer and it perform Broadcasting for any received signal means that regeneration, for example a network consisted of two switches (1 and 2) and one router, where host (A) is connected to switch 1 , host $(\mathrm{B})$ is connected to switch 2 and host (C) is connected to same network but through the router, we find that host A and B can see themselves through their switches because of the two switches are layer two so host A and B can distinguish each other through layer two, but host C can't see the other hosts because it is connected to the network through the router, where it understands layer three protocol (IP) not MAC address of data link layer (layer two).

\subsubsection{Bridge}

Is a Layer two device, but it can't understand the MAC address; it can reduce the number of collision domains. 


\begin{tabular}{|c|c|c|}
\hline MODOT & $\begin{array}{c}\text { Mssouri Department of Transportation } \\
\text { Transportation Planning } \\
\text { Fatal Crashes by Drivers Age for } 2009 \\
880 \text { Drivers involved In } \\
577 \text { Fatal Crashes }\end{array}$ & 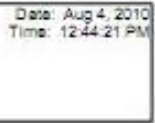 \\
\hline
\end{tabular}

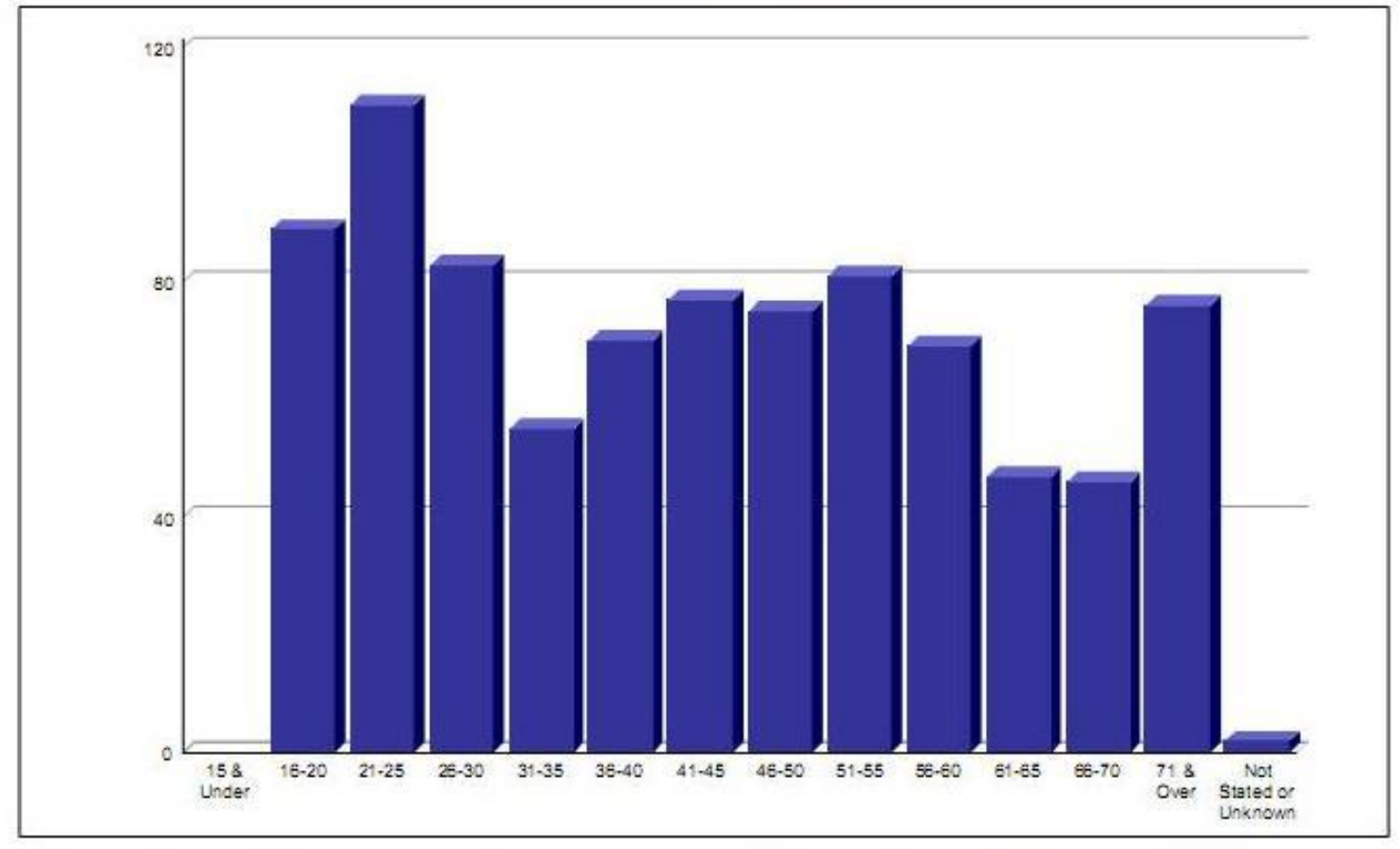

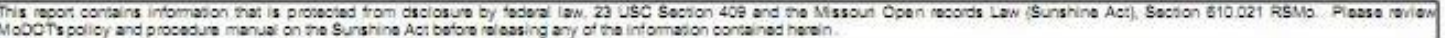

Figure 1. Missouri Department of Transportation, Transportation Planning Fatal Crashes by Drivers Age for 2009880 Drivers Involved In 577 Fatal Crashes

\subsubsection{Router}

Is a Layer Three (Network Layer) device, it can understand frame but neither MAC address nor bit streaming of Physical layer, where each router in a wide network or group of LANs are connected to each other establish a routing table contain all Hosts IPs in its LAN then all routers exchanges all these tables so according to it any host acts the best path to the wanted destination through different routers such in figure, where the router interface that front of other network is called Gateway.

\subsubsection{Transmission Media}

Is a media to carry Packet Data unit (PDU), which travel certain distance by the signal speed and also has capacity that can't be exceeded.

\subsection{Data Network Performance ${ }^{[7]}$.}

This category is divided into two points, firstly the delay types as in the following.

\subsubsection{Transmission Delay}

The consumed time till final bit leaves the transmitter.

\subsubsection{Propagation Delay}

The consumed time within the data transfers from the transmitter to the receiver.

\subsubsection{Queuing delay}

The produced time about the receiver buffer is fully occupied by data to be processed.

\subsubsection{Processing delay}

The time consumed during data processing.

\subsubsection{Reassembly delay}

The consumed time for reconstructing a received data that is assembled before at a transmitter.

Secondary utilization, it is easy to be obtained by the different used media access technique such that (ALOHA, SLOTED ALOHA, CSMA, CSMA/CD) where the best of them is CSMA/CD.

\subsection{Data Network Problems}

losses according to exceeding the time out that is determined for incoming data in specific network, errors means that converting certain bit or stream of bits from ' 0 ' to ' 1 ' and vice versa where real time application (Voice or Video) can imply or suffer error but not delay and vice versa proportional to data.

\section{WHY MAPPING BETWEEN DATA NETWORKS AND ROAD NETWORKS?}

The data network is a fast growing technology, many resources study the data network problems and provided solutions where 
as the grows of road network problem solutions are very slow compared to the data network solutions, the main purpose is to get the advantages of data network solutions and look for those applications to the road network to improve its performance, according to the road network crisis and congestion there are some solutions are exhibited such the following ${ }^{[2]}$ :

- Adaptive Cruise Control (ACC), similar to sensor network [3].

- Lane Keeping Support (LKS), similar to sensor network [4].

- $\quad$ Advanced Driver Assistance Systems (ADAS)

- $\quad$ Automated Highway Systems (AHS)

- $\quad$ Autonomous Intelligent Cruise Control (AICC)

- Cooperative Intelligent Vehicle-Highway Systems (CIVHS

- Intelligent Cruise Control (ICC)

- $\quad$ Lane Departure Avoidance (LDA)

- $\quad$ Lane Departure Warning Assistant (LDWA)

- Autonomous Intelligent Cruise Control (AICC)

- $\quad$ Lane Keeping Support (LKS)

- $\quad$ Advanced Vehicle Control Systems (AVCS)

- $\quad$ Autonomous Speed Assistant (ASA)

- National Automated High Way System Consortium (NAHSC)

Autonomous (Adaptive) cruise control is an optional cruise control system appearing on some more upscale vehicles. The system goes under many different trade names according to the manufacturer. These systems use either a radar or laser setup allowing the vehicle to slow when approaching another vehicle and accelerate again to the preset speed when traffic allows.

Lane keeping support (LKS) systems aim at assisting the driver to keep the vehicle safely in the own lane. They utilize visionbased sensing to observe the road curvature and the host vehicle's position in lane.

Let's know a brief description for some mapped parameters in the above tables.

\subsection{Road vs. Transmission media and Buffer}

Distance can be represented in both of them as distance according to dealing with longitudinal dimension for the two systems (the road length is corresponding to the cable length). Speed compared to the signal speed with constraining on the propagation delay due to time consumption that exhausted in transferring some sort of packets (message) from specific node to other, so it can be matched to vehicles speed. Lanes compared to parallel transmission or not, where the parallel transmission mayn't be applicable because vehicles can change their lanes but data parallel transmission can't. Vehicle delay on the road from one point to another can be compared to the propagation delay which is defined as the time that the signal takes from source to the destination through the network cable. Network delays can be divided into five categories: the transmission delay is the consumed time of leaving final bit of certain message from the transmitter, propagation delay is the consumed time for reaching the data from the source to the destination through the link between them, queuing delay is the consumed time due to waiting the incoming data in the buffer because of processing for the current data at the processor on the other hand proportional to vehicles the queuing delay represent waiting in certain stop sign or traffic light, processing delay is the consumed time to process the current data in the processor and reassembly delay is the consumed time for assembling the incoming PDUs at the receiver after dividing them at the transmitter according to the receiver ready. Merge can be compared to carrier sense multiple access (CSMA) type of media access technique because of the merging of two ways to one way need to entry conditions from both ways depending on some roles and laws that can be represented by the media access technique. Vehicles accident that affect to the vehicles themselves can't be compared to PDU collision, because vehicle's accident causes road jam, tremendous slow down, and high delay. Whereas PDU collision only cause loss of the collide PDUs. Cross-section can be represented by two types, first as switch, where it receives the data from one port and sends to another port and the second as add drop multiplexer in case of vehicles gathering in same direction. Road map compared to topology, where we have several types of topologies such that mesh means dedicated paths, star, bus, ring, tree and hybrid topology.

Table 1. Road vs. Transmission media and Buffer

\begin{tabular}{|c|c|c|}
\hline Road & $\begin{array}{c}\text { Tx. Media \& } \\
\text { Buffer }\end{array}$ & Solution \\
\hline Distance. & Distance. & Matched \\
\hline Speed. & $\begin{array}{l}\text { Signal Speed } \\
\text { (Propagation } \\
\text { Delay). }\end{array}$ & Matched \\
\hline Lanes. & $\begin{array}{l}\text { N/A or Parallel } \\
\text { transmission, } \\
\text { where the parallel } \\
\text { transmission. May } \\
\text { be not applicable, } \\
\text { because vehicles } \\
\text { can change their } \\
\text { lanes but the } \\
\text { parallel } \\
\text { transmitted data } \\
\text { can't. }\end{array}$ & Matched \\
\hline Delay & Delay & $\begin{array}{l}\text { Propagation delay of road } \\
\text { Can be considered as } \\
\text { processor processing delay, } \\
\text { where the delay types: }\end{array}$ \\
\hline
\end{tabular}




\begin{tabular}{|c|c|c|}
\hline & & $\begin{array}{ll}\text { - } & \text { Tx. Delay. } \\
\text { - } & \text { Propagation } \\
& \text { Delay. } \\
\text { - } & \text { Queuing delay. } \\
\text { - } & \text { Processing delay. } \\
\text { - } & \text { Reassembly } \\
& \text { delay. }\end{array}$ \\
\hline $\begin{array}{l}\text { Change } \\
\text { Lane. }\end{array}$ & No Change & Matched \\
\hline $\begin{array}{l}\text { Variable } \\
\text { Speed. }\end{array}$ & Constant Speed & Av. Speed \\
\hline Merge & $\begin{array}{l}\text { Media Access } \\
\text { Technique }\end{array}$ & $\begin{array}{l}\text { (CSMA), because it not } \\
\text { guarantee the collision such } \\
\text { the Merge. }\end{array}$ \\
\hline Accident & Collision & Not matched \\
\hline $\begin{array}{l}\text { Accident } \\
\text { recovery }\end{array}$ & $\begin{array}{l}\text { Collision } \\
\text { (Retransmission) }\end{array}$ & Matched \\
\hline $\begin{array}{l}\text { Affect to } \\
\text { incoming } \\
\text { Vehicles }\end{array}$ & $\begin{array}{l}\text { Doesn't affect to } \\
\text { incoming packets }\end{array}$ & Matched \\
\hline Bridge & $\begin{array}{l}\text { Link (as shortcut } \\
\text { controlled } \quad \text { by } \\
\text { roles) }\end{array}$ & Matched \\
\hline Tunnel & $\begin{array}{l}\text { Link (as shortcut } \\
\text { controlled } \quad \text { by } \\
\text { roles) }\end{array}$ & Matched \\
\hline U-Turn & $\begin{array}{l}\text { N/A (or partially } \\
\text { loop back) }\end{array}$ & Matched \\
\hline $\begin{array}{l}\text { Cross- } \\
\text { Sections }\end{array}$ & $\begin{array}{l}\text { Switch, Router or } \\
\text { add drop } \\
\text { multiplexer. }\end{array}$ & Matched \\
\hline Splitting & $\begin{array}{l}\text { Add } \\
\text { Multiplexing, } \\
\text { DeMux (but not } \\
\text { equal weight } \\
\text { because not } \\
\text { should all } \\
\text { branched vehicles } \\
\text { the same) or } \\
\text { Switching. }\end{array}$ & Matched \\
\hline Road Map & Topology & Matched \\
\hline $\begin{array}{l}\text { Queuing } \\
\text { Delay }\end{array}$ & Queuing Delay & Matched \\
\hline
\end{tabular}

\subsection{Toll station vs. Processor}

In the toll station entry there is a distance that represents a buffer for the vehicles which wait in a queue to pay the toll and then move forward after that, in the data network the packets which arrive to a network element Wait in its memory in a queue waiting to be processed, there are other similarities between these two models like the used queuing model (for example, First In First Out, priority, buffer size, ...etc.).

Table 2. Toll station vs. Processor

\begin{tabular}{|l|l|l|}
\hline \multicolumn{2}{|c|}{ Toll Station } & \multicolumn{1}{c|}{ Polution } \\
\hline $\begin{array}{l}\text { Distance (road) } \\
\text { before the toll } \\
\text { station }\end{array}$ & Buffer & Matched \\
\hline Speed. & Speed (Low) & Matched \\
\hline Booth & Parallel processing & Matched \\
\hline $\begin{array}{l}\text { Special type Lanes } \\
\text { toll booth) }\end{array}$ & $\begin{array}{l}\text { Specifying the data } \\
\text { type processing }\end{array}$ & Matched \\
\hline Change regions & Change sub network & Matched \\
\hline Paying toll & Processing Delay & Matched \\
\hline Queuing & Queuing Delay & Matched \\
\hline
\end{tabular}

\subsection{Traffic light and road vs. switch}

The stop sign (red) of traffic light causes waiting vehicles in form queue till the determined time of the stop sign end to can leave this operation can be represented as vehicles register so it can be matched with waiting packets inside a buffer till finishing the previous packets to be processed and then these packet enter to be processed too so it is the same concept proportional to the inter arrival time. According to the delay that the data suffers during data processing, and then this data will follow to be propagated again, it is similar to the vehicles waiting inside a traffic light to move again means that recovers its speed.

Table 3. Traffic light and road vs. switch

\begin{tabular}{|l|l|l|}
\multicolumn{1}{|c|}{$\begin{array}{c}\text { Traffic Light \& } \\
\text { Road }\end{array}$} & \multicolumn{1}{|c|}{ Switch } & \multicolumn{1}{|c|}{ Solution } \\
\hline $\begin{array}{l}\text { Distance before the } \\
\text { traffic light }\end{array}$ & Buffer & Matched \\
\hline $\begin{array}{l}\text { Vehicle inter } \\
\text { arrival time }\end{array}$ & $\begin{array}{l}\text { Packet inter arrival } \\
\text { time }\end{array}$ & Matched \\
\hline $\begin{array}{l}\text { Road } \\
\text { (traditional Way \& } \\
\text { High way) }\end{array}$ & $\begin{array}{l}\text { Type } \\
\text { Ethernet) }\end{array}$ & Matched \\
\hline Distribution Paths & Ports (Branches) & Matched \\
\hline
\end{tabular}




\begin{tabular}{|l|l|l|}
\hline Speed Recovery & $\begin{array}{l}\text { Due to the processor } \\
\text { processing delay }\end{array}$ & Matched \\
\hline Traversing Delay & Transmission Delay & Matched \\
\hline
\end{tabular}

\subsection{Traffic Light vs. switching}

Virtual circuit switch such that asynchronous transmission mode that drive the PDU (cell) to move sequentially like the train trucks, so it is compared to the train. Packet switching uses dedicated path for the PDU but this path can be used for another host such data gram, so it can be compared to vehicles that pass through the traffic light. The train bars are the determined paths of the train to move, according to specific direction or trip, so it is compared to circuit switching in case of data network, where the data moves in dedicated path without any alternative paths at occurring failure, so it is the same concept proportional to Emergency Vehicles Lanes vs. Circuit Switching.

Table 4. Traffic Light vs. switching

\begin{tabular}{|l|l|l|}
\multicolumn{1}{|c|}{ Traffic light } & \multicolumn{1}{|c|}{ Switching } & \multicolumn{1}{c|}{ Solution } \\
\hline Train bars & Circuit Switching & Matched \\
\hline $\begin{array}{l}\text { Emergency } \\
\text { Vehicles Lanes }\end{array}$ & $\begin{array}{l}\text { Virtual Path } \\
\text { Network (VPN) }\end{array}$ & Matched \\
\hline Trains & $\begin{array}{l}\text { Virtual Circuit } \\
\text { Switching (ATM) }\end{array}$ & Matched \\
\hline Vehicles & $\begin{array}{l}\text { Packet Switching } \\
\text { (Data gram) }\end{array}$ & Matched \\
\hline
\end{tabular}

\subsection{Vehicles vs. PDU}

Real time PDU can be compared to emergency vehicles, where the real time PDU such that audio or video where they use the UDP protocol. Special Lanes are reserved lanes for certain type of vehicles like Ambulance vehicles, fire extinguisher vehicles ...etc, so it can be matched with special format of PDU because of the distinguished shape and use, so it is the same concept proportional to Special Size vs. Special priority of Processing.

Table 5. Vehicles vs. PDU

\begin{tabular}{|l|l|l|}
\hline \multicolumn{1}{|c|}{ Vehicle } & \multicolumn{1}{|c|}{ PDU } & \multicolumn{1}{c|}{ Solution } \\
\hline $\begin{array}{l}\text { Emergency } \\
\text { Vehicles }\end{array}$ & Real Time PDU & Matched \\
\hline
\end{tabular}

\begin{tabular}{|l|l|l|}
\hline Special Lanes & Special format & Matched \\
\hline Special Size & $\begin{array}{l}\text { Special Priority of } \\
\text { Processing }\end{array}$ & Matched \\
\hline $\begin{array}{l}\text { Non Emergency } \\
\text { Vehicles }\end{array}$ & Non real time PDU & Matched \\
\hline Vehicles Size & PDU Length & Matched \\
\hline $\begin{array}{l}\text { Vehicles Speed } \\
\text { (m/s) }\end{array}$ & $\begin{array}{l}\text { PDU Speed (Signal } \\
\text { Speed) }\end{array}$ & Matched \\
\hline \# of Vehicles & \# of PDUs & Matched \\
\hline Delaying & Delaying & Matched \\
\hline $\begin{array}{l}\text { Vehicle } \\
\text { arrival } \\
\text { distribution) }\end{array}$ & $\begin{array}{l}\text { PDU Source (PDU } \\
\text { generation } \\
\text { Distribution) }\end{array}$ & Matched \\
\hline $\begin{array}{l}\text { Vehicle Destination } \\
\text { Roads }\end{array}$ & PDU Destination & Matched \\
\hline N/A & Transmission Media & Matched \\
\hline
\end{tabular}

\subsection{Road vs. Router}

Branches and shortcuts (Signs) of the road network are used to serve the vehicles identify the paths and directions so it is matched to Processor factors in addition to determining paths at routing for PDU.

Table 6. Road vs. Router

\begin{tabular}{|l|l|l|}
\hline \multicolumn{1}{|c|}{ Road } & \multicolumn{1}{|c|}{ Router } & \multicolumn{1}{c|}{ Solution } \\
\hline $\begin{array}{l}\text { Branches and } \\
\text { shortcuts (Signs) }\end{array}$ & $\begin{array}{l}\text { Processor factors in } \\
\text { addition to } \\
\text { determining paths at } \\
\text { routing }\end{array}$ & Matched \\
\hline Vehicles & IP Packet & Matched \\
\hline Roles & Protocols & Matched \\
\hline $\begin{array}{l}\text { Distance between } \\
\text { Vehicles }\end{array}$ & $\begin{array}{l}\text { Enter arriving time } \\
\text { (put layer 3 specs) }\end{array}$ & Matched \\
\hline
\end{tabular}



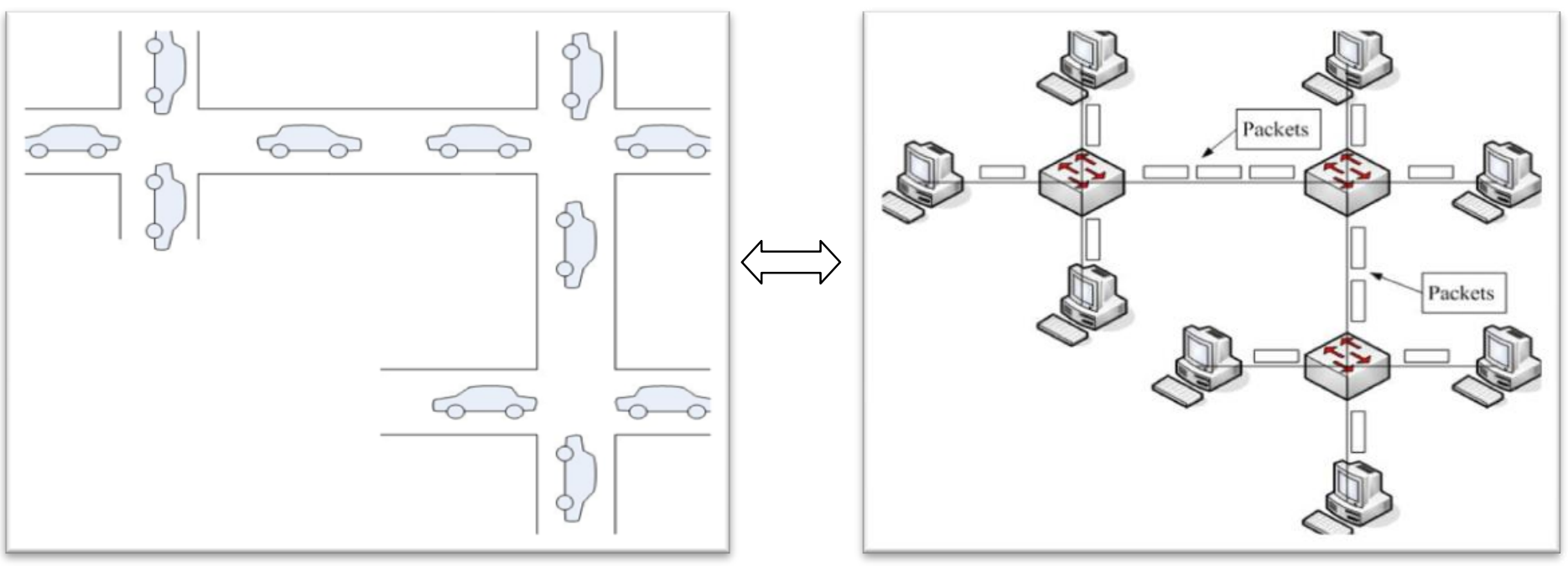

Figure 2. Matching between Switch device to multiple realities such traffic light, stop sign, cross-section and toll booth

Let's discuss two examples depending on above table, firstly Switch, this device can be compared to multiple realities such traffic light, stop sign, cross-section and toll booth (station); where we can obtain in figure 2.

Secondary real Queuing of the road network can be compared by data network queuing where it is supported some analysis such $\mathrm{M} / \mathrm{M} / 1, \mathrm{M} / \mathrm{M} / \mathrm{D}$ and $\mathrm{M} / \mathrm{M} / \mathrm{G}$ queue also the data network provided solutions to reduce the queue and speed up the flow such that rapid processing using multiple processors, controlling the memory and giving priority ${ }^{[8]}$.

\section{CONCLUSION}

Recently according to the data network solutions prosperity and appearing multiple simulation programs that facilitate the

mission of getting any model for solving real problem, so after performing own model we can achieve excellent results and statistics may be crystallized for the road network according to the mapping table and represent the real traffic, therefore it is easy to get a fast solution for the road network under the traffic crises in the different world areas.

\section{REFERENCES}

[1] Techniques: a research proposal, Papers of the 25th Conference of Australian Institutes of Transportation Research, Adelaide, ogiatzis, N Since 2003

[2] Road Networks of the Future: can Data Networks Help?, Paper for Australasian Transport Research Forum, Sydney, Since 2005.

[3] Adaptive Cruise Control System Overview 5th Meeting of the U.S. Software System Safety Working Group April 12th-14th 2005 @ Anaheim, California USA.

[4] Mauro Montiglio, Stefania Martini, Vincenzo Murdocco DEVELOPMENT OF A LANE KEEPING SUPPORT SYSTEM FOR HEAVY-TRUCKS Centro Ricerche Fiat ScpA - Strada Torino 50, 10043 Orbassano (TO), Italy
[5] Traffic Crash Statistics, Missouri Department of Transportation, 2009 Missouri State high Way System.

[6] LOCAL AND METROPOLITAN AREA NETWORKS, WILLIAM STALLING, prentice Hall Inc., Upper Saddle River, New Jersey Since 2000,1997.

[7] DATA AND COMPUTER COMMUNICATIONS, WILLIAM STALLING, prentice Hall Inc., Upper Saddle River, New Jersey Since 2007.

[8] Fundamentals of Telecommunication Networks By Tarek N. Saadaw , Mostafa H. Ammar and Ahmed El Hakeems.

[9] Objects and components in Locality-Scope, Papers of the Intelligent Vehicles and Road Infrastructure Conference, Melbourne, Vogiatzis, N Since 2005

[10] Traffic Matrix Inference in IP Networks, in 10th International Telecommunication Network Planning Symposium, http://perso.rd.francetelecom.fr/roberts/Pub/BR02.pdf, Benameur, N and Roberts, J W Since 2002

[11] Challenges of Inter-Vehicle Ad Hoc Networks, IEEE Transactions on Intelligent Transportation Systems, Blum, J; Eskandarian, A and Hoffman, L Since 2004

[12] The Statistics of Dynamic Networks, PhD Thesis, Department of Mathematics, University of York, Clegg, R G Since 2004

[13] Modelling the SPA system for increased efficiency of signalised intersections using ITS vehicle control technologies, PhD thesis, Department of Information Technology, Engineering and the Environment: University of South Australia, Adelaide, Clement, S J Since 2003

[14] Evaluation of lane changing and merging in microsimulation models, Papers of the $27^{\text {th }}$ Australasian Transport Research Forum (27), Adelaide, Hidas, P Since 2005 
[15] Abdel Karim Al Tamimi, Chakchai So-In, Raj Jain, "Modeling and Resource Allocation for Mobile Video over WiMAX Broadband Wireless Networks," IEEE Journal on Selected Areas in Communications, Special issue on Wireless Video Transmission, April 2010, Vol. 28, No. 3, pp.354-365,

http://www1.cse.wustl.edu/ jain/papers/vid_jsac.htm

[16] Subharthi Paul, Jianli Pan, Raj Jain, "Architectures for the Future Networks and the Next Generation Internet: A Survey," Computer Communications, UK, Volume 34, Issue 1, 15 January 2011, Pages 2-42, http://www1.cse.wustl.edu/ jain/papers/i3survey.htm

[17] Abdel Karim Al Tamimi, Raj Jain, Chakchai So-In, "Dynamic Resource Allocation Based on Online Traffic Prediction for Video Streams," 4th IEEE International Conference on Internet Multimedia Systems Architecture and Application (IMSAA) 2010, Bangalore, India, December $15-18$, 2010 ,

http://www1.cse.wustl.edu/ jain/papers/imsaa10.htm

[18] Abdel Karim Al Tamimi, Raj Jain, Chakchai So-In, "Statistical Analysis and Modeling of High-Definition Video Traces," Proceedings of IEEE International Conference on Multimedia anad Expo (ICME) 2010, Singapore, July 19-23, 2010, http://www1.cse.wustl.edu/ jain/papers/icme10.htm

[19] U. Demiryurek, F. B. Kashani, and C. Shahabi. Efficient continuous nearest neighbor query in spatial networks using euclidean restriction. In SSTD, Since 2009

[20] Mate Boban, Tiago T. V. Vinhoza, Modeling and Simulation of Vehicular Networks: Towards Realistic and Efficient Models, IEEE Wireless Communications and Networking Conference since January 2011. 